\title{
A basal cell defect promotes budding of prostatic intraepithelial neoplasia
}

\author{
Mengdie Wang ${ }^{1}$, Raymond B. Nagle ${ }^{2}$, Beatrice S. Knudsen ${ }^{3}$, Gregory C. Rogers ${ }^{1, *}$ and Anne E. Cress ${ }^{1, *}$
}

\begin{abstract}
Basal cells in a simple secretory epithelium adhere to the extracellular matrix (ECM), providing contextual cues for ordered repopulation of the luminal cell layer. Early high-grade prostatic intraepithelial neoplasia (HG-PIN) tissue has enlarged nuclei and nucleoli, luminal layer expansion and genomic instability. Additional HG-PIN markers include loss of $\alpha 6 \beta 4$ integrin or its ligand laminin-332, and budding of tumor clusters into laminin-511-rich stroma. We modeled the invasive budding phenotype by reducing expression of $\alpha 6 \beta 4$ integrin in spheroids formed from two normal human stable isogenic prostate epithelial cell lines (RWPE-1 and PrEC 11220). These normal cells continuously spun in culture, forming multicellular spheroids containing an outer laminin-332 layer, basal cells (expressing $\alpha 6 \beta 4$ integrin, high-molecular-weight cytokeratin and p63, also known as TP63) and luminal cells that secrete PSA (also known as KLK3). Basal cells were optimally positioned relative to the laminin-332 layer as determined by spindle orientation. $\beta 4$-integrindefective spheroids contained a discontinuous laminin-332 layer corresponding to regions of abnormal budding. This 3D model can be readily used to study mechanisms that disrupt laminin-332 continuity, for example, defects in the essential adhesion receptor ( $\beta 4$ integrin), laminin-332 or abnormal luminal expansion during HG-PIN progression.
\end{abstract}

\section{KEY WORDS: Prostate, Neoplasia, Integrin, Laminin, Spheroids}

\section{INTRODUCTION}

The normal prostate gland is a simple secretory epithelium containing a basal cell population [which can be detected by analyzing for the presence of high-molecular-weight cytokeratin (HMWCK)] harboring stem cells (Bonkhoff, 1996; Bonkhoff and Remberger, 1996; Bostwick, 1996a,b) and a luminal cell population [detected by racemase (AMACR) staining] that secretes PSA (also known as KLK3) (Thomson and Marker, 2006). During normal glandular development, extracellular matrix (ECM)-cell-receptor interaction provides contextual cues and a developmental 'morphogenesis checkpoint' for ordered repopulation (Brown, 2011). Cell divisions parallel to the basal cell surface maintain proximity to the ECM and control mitotic spindle orientation during epithelial morphogenesis and repair (Xia et al., 2015).

\footnotetext{
'Department of Cellular and Molecular Medicine, College of Medicine, University of Arizona Cancer Center, Tucson, AZ 85724, USA. ²Department of Pathology, College of Medicine, University of Arizona Cancer Center, Tucson, AZ 85724, USA ${ }^{3}$ Department of Pathology and Laboratory Medicine, Cedars Sinai Medical Center, Los Angeles, CA 90048, USA.

*Authors for correspondence (gcrogers@email.arizona.edu; cress@email.arizona.edu)

D A.E.C., 0000-0001-6194-0061
}

Received 16 February 2016; Accepted 2 September 2016
During the early stages of prostate cancer progression, a defective glandular structure forms, called high-grade prostatic intraepithelial neoplasia (HG-PIN), which is defined by focal loss or attenuation of the basal cell layer and ECM (Nagle et al., 1994), and loss of both integrin $\alpha 6 \beta 4$ expression and its the corresponding ECM ligand laminin-332 (i.e. laminin comprising the $\alpha 3 \mathrm{~A} \beta 3 \gamma 2$ chains) (Cress et al., 1995; Davis et al., 2001; Hao et al., 1996; Nagle et al., 1995; Pontes-Junior et al., 2009). HGPIN often contains a mixture of basal and luminal cell markers, consistent with a loss of normal contextual cues and mitotic spindle misorientation (Bonkhoff and Remberger, 1996) as seen during regrowth of prostate following castration and androgen reintroduction (Verhagen et al., 1988). HG-PIN has genomic instability (Haffner et al., 2016; Iwata et al., 2010; Mosquera et al., 2009, 2008; Nagle et al., 1992; Petein et al., 1991) and is a precursor of invasive prostate cancer (Bonkhoff and Remberger, 1996; Bostwick, 1996a,b; Bostwick et al., 1996; Haggman et al., 1997; Montironi et al., 1996a,b; Montironi and Schulman, 1996).

Here, we report a new three-dimensional (3D) HG-PIN-type model using two different isogenic human prostate epithelial cell lines, called RWPE-1 (Bello et al., 1997; Roh et al., 2008; Webber et al., 1997) and PrEC 11220, with a stable modification to deplete $\beta 4$ integrin expression. The model provides a means to test consequences of basal cell defects in human HG-PIN progression.

\section{RESULTS AND DISCUSSION Human HG-PIN in tissue and the absence of $\alpha 6 \beta 4$ integrin expression}

Normal human prostate glands contain an ordered basal and luminal cell distribution, as shown in Fig. 1A,B. Integrin $\alpha 6 \beta 4$ is found within the basal cell layer (Fig. 1C) and is required for anchoring basal cells to laminin-332 ECM through the hemidesmosome (Nagle et al., 1995; Pulkkinen and Uitto, 1998; Wilhelmsen et al., 2006). In contrast, HG-PIN (Fig. 1 A,B) contains cells with enlarged nuclei and prominent nucleoli that proliferate within the lumen, enlarging the glands, resulting in continuity gaps (Nagle and Cress, 2011). In these gaps, laminin-332 and $\alpha 6 \beta 4$ integrin - essential requirements for functional hemidesmosomes - are absent; HG-PIN and cancer lesions are known to lack basal cells and laminin-332 deposition, becoming exposed to laminin-511 (i.e. laminin comprising the $\alpha 5 \beta 1 \gamma 1$ chains) within the muscle stroma (Davis et al., 2001; Nagle and Cress, 2011; Nagle et al., 1995). Defective $\beta 4$ integrin function results in defective laminin-332 assembly (Yurchenco, 2015) and laminin-511 is a known potent morphogen essential for embryonic development (Ekblom et al., 1998). We observed extensive budding of cell clusters through $\beta 4$ integrin gaps and into the stroma in HG-PIN (Fig. 1D, asterisk). Hence, loss of $\alpha 6 \beta 4$ integrin is associated with abnormal outgrowth of the epithelium in human HG-PIN. 
A

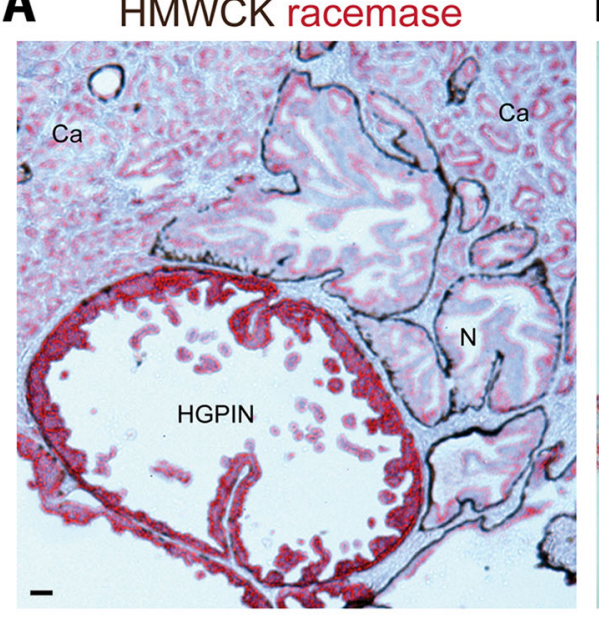

C

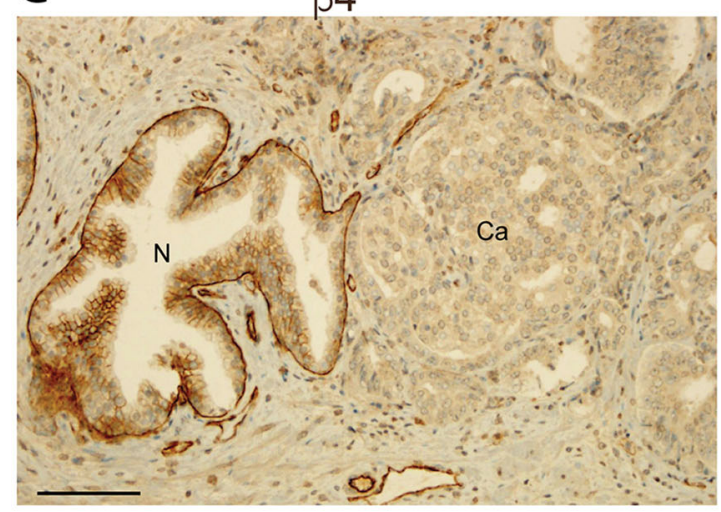

B

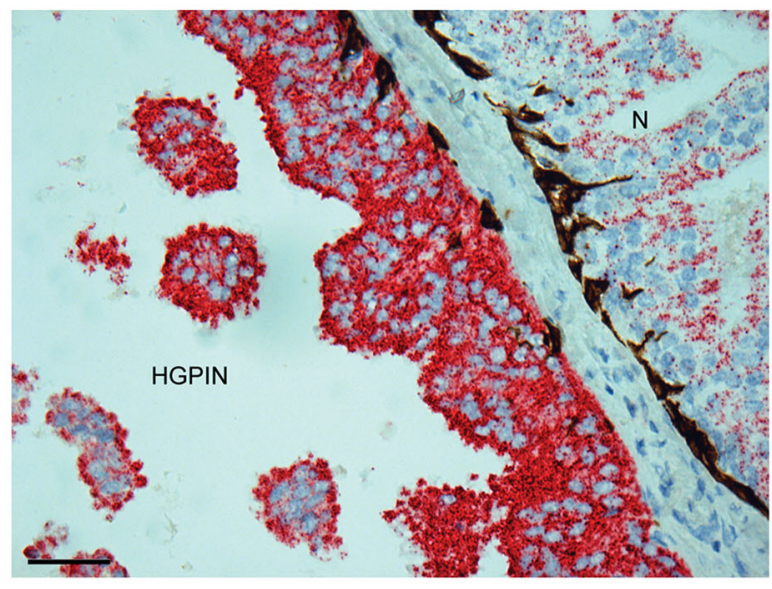

D

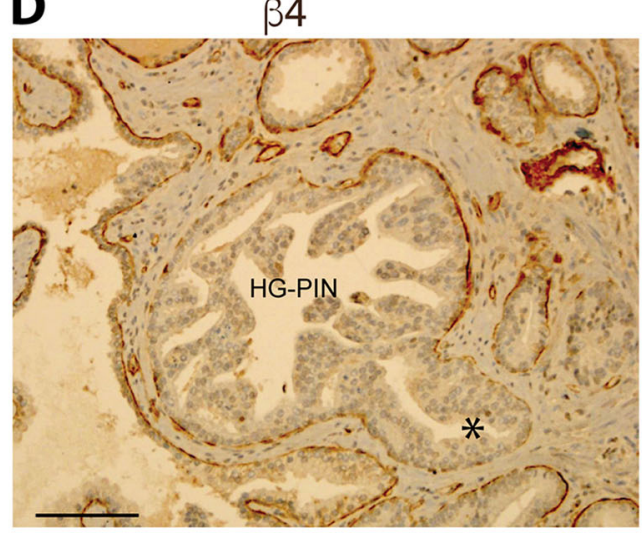

Fig. 1. Human HG-PIN in tissue and the focal absence of $\alpha 6 \beta 4$ integrin expression. (A,B) Human prostate tissue was stained for HMWCK (brown stain) to mark basal cells and $\alpha$-methylacyl CoA racemase (P504S, red stain) to mark luminal cells (Kumaresan et al., 2010). (A) Representative image showing

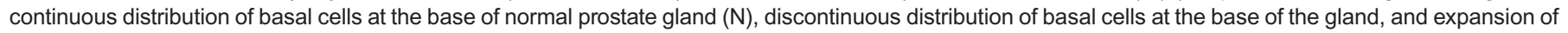
cells into the lumen in high-grade PIN (HG-PIN) and prostate carcinoma (Ca). Note the loss of basal cell layer in cancer. (B) Higher magnification of region in A

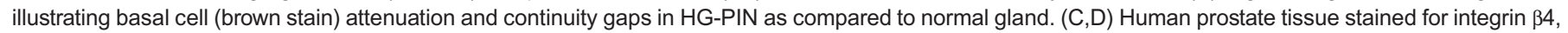
showing basal cell distribution as in A and B. Note the budding of HG-PIN into muscle stroma (asterisk). Scale bars: $100 \mu \mathrm{m}$.

\section{A 3D model for prostate glands contains basal and luminal cell architecture}

Given the importance of the glandular structure for maintaining homeostasis (Brown, 2011; Chandramouly et al., 2007; Wilhelmsen et al., 2006; Xia et al., 2015) and the role of the basal cell stem cell compartment (Bonkhoff and Remberger, 1996; Bostwick, 1996b; Hudson, 2004; Karthaus et al., 2014; Schmelz et al., 2005), we generated a 3D model of prostate glands using two different primarily diploid prostate epithelial cell lines, RWPE-1 and PrEC 11220. RWPE-1 is a stable non-tumorigenic human cell line derived from a histologically normal adult human prostate (Bello et al., 1997; Roh et al., 2008; Webber et al., 1997) and is used as a normal prostate cell line in genomic and differentiation studies (Wang et al., 2011; Webber et al., 1997). We note that RWPE-1 was isolated from a donor undergoing cryoprostatectomy and might carry abnormalities. The cell line was immortalized by HPV-18 and has characteristics of an intermediate cell type (Verhagen et al., 1992) expressing both basal and luminal cell cytokeratin, androgen receptor and PSA in response to androgen (Bello et al., 1997). For comparison to RWPE-1 cells, we used another normal human prostate cell line (PrEC 11220), immortalized by hTERT, to test spheroid formation (Dalrymple et al., 2005; Salmon et al., 2000). We found both cell lines formed compact multicellular spheroids as represented by RWPE-1 (Fig. 2A) and observed a dramatic cellspinning phenotype as spheroids formed from a single cell (Movies 1 and 2). The rotational motion was similar to mammary epithelial acini that coordinate rotational movement with laminin matrix assembly (Wang et al., 2013). Most (65\%) of the RWPE-1 spheroids contained a lumen after 10 days, as measured by presence of $\beta$-catenin on the cell surface and absence of DAPI staining nuclei in the interstices of the spheroid (Fig. 2B,C). Positive detection of four different molecular markers [laminin-332 production and organization, $\alpha 6 \beta 4$ integrin expression, HMWCK and p63 (also known as TP63)] confirmed the presence of basal cells within spheroids created by both RWPE-1 and PrEC 11220 cell lines (Fig. 2D). Spheroid production was seen in both cell lines and, hence, was independent of the immortalization method. The RWPE-1 cells were superior in forming a continuous and wellcircumscribed layer of laminin-332 and continuous $\beta 4$ integrin distribution as compared to the PrEC 11220 cells (Fig. 2D). The PrEC 11220 cells contained a punctate yet circumscribed distribution of $\beta 4$ integrin, consistent with the dynamic processes expected when the basal lamina forms as directed by $\beta 4$ integrin clustering on basal cells (Yurchenco, 2015). Taken together, the data suggest that both cell lines assemble laminin-332 and $\beta 4$ integrin on the spheroid surface. The punctate distribution observed 


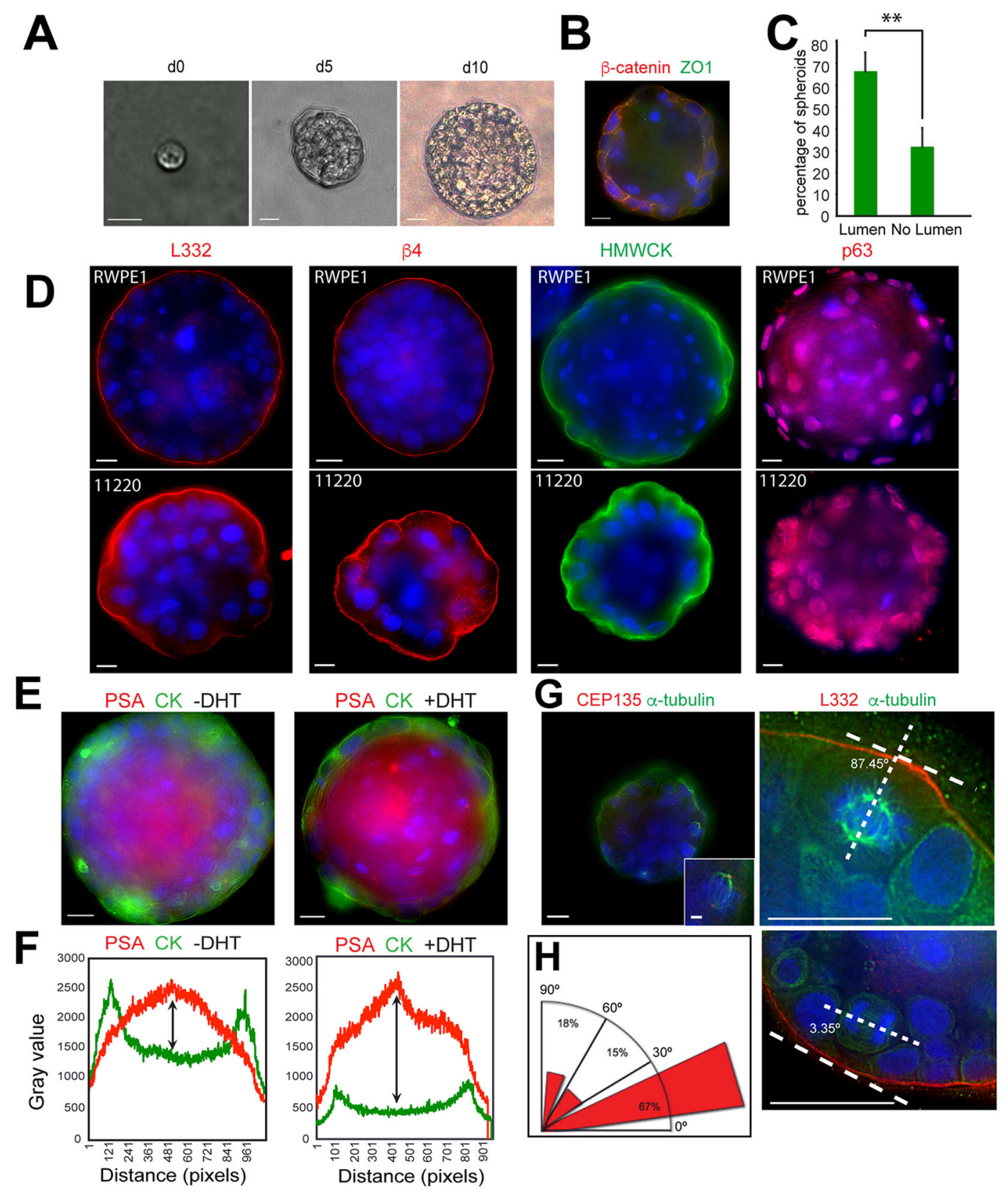

Fig. 2. 3D morphogenesis of PIN arising from single cells. (A) RWPE-1 acini growth from a single cell after the indicated number of days (d) in culture. Scale bars: $20 \mu \mathrm{m}$. (B) A day-14 RWPE-1 spheroid stained for $\beta$-catenin (red), and ZO1 (green). Scale bar: $10 \mu \mathrm{m}$. (C) Quantification of the number of RWPE-1 spheroids with lumens on day 10. Data are mean \pm s.d. from three experiments, $n=100 .{ }^{* *} P=0.0088$ (two-tailed $t$-test). (D) Spheroids from RWPE-1 cells on day 14 (top row) and PREC 11220 cells on day 10 (bottom row) stained for with laminin-332 (L332, red), integrin $\beta 4$ (red), HMWCK (green) and p63 (red). Scale bars: $10 \mu \mathrm{m}$. (E) Day-14 RWPE-1 spheroids without DHT (left) and with DHT (right) stained for cytokeratin 5 and 14 (CK, green) and PSA (red). Scale bars: $10 \mu \mathrm{m}$. (F) DHT treatment induces a twofold increase in PSA production as determined by a quantification of signal intensity for cytokeratin 5 and 14 distribution (green) and PSA distribution (red) across the images in E. The PSA intensity signal relative to cytokeratin signal is indicated by the size of double-headed arrow.

(G) Single-plane images of day-10 spheroids. Staining for CEP135 (left panel, red) and $\alpha$-tubulin (left panel, green) reveals the spindle orientation. The right-hand panel shows spheroids stained for $\alpha$-tubulin (green), laminin-332 (red) and DNA (blue) to highlight spindle orientation relative to laminin-332. Note that cell division parallel to basement membrane (laminin-332) results in two cells that are located side by side, whereas cell division perpendicular to laminin-332 results in cells located in the lumen. Scale bars: $10 \mu \mathrm{m}$ (main images), $1 \mu \mathrm{m}$ (inset in top left). (H) Radial histograms (rose plots) showing mitotic spindle angle relative to the laminin-332 layer of dividing cells in RWPE-1 spheroids on day 10; $n=33$ spindles. Nuclei in all panels were stained with Hoechst 33342 (blue).

with PrEC 11220 spheroids suggests that it could be useful for tracking dynamic $\beta 4$-integrin-directed assembly of the basal lamina. In contrast, RWPE-1 spheroids assembled a robust and tight laminin-332 and $\beta 4$ integrin layer, which could be used as a stringent test for determining the influence of contextual signals on the invasive budding in HG-PIN. RWPE-1 spheroids contained functional luminal cells as increased PSA secretion could be detected in response to dihydrotestosterone (DHT) treatment compared to untreated controls (Fig. 2E,F).

We next measured the accuracy of mitotic spindle orientation in basal cells from RWPE-1 spheroids. The RWPE-1 spheroids were used as these have a highly ordered laminin-332 layer as a reference point. Mitotic spindles and spindle poles were visualized by staining for $\alpha$-tubulin and the centriole protein CEP135; spindle orientation 
in basal cells was measured relative to laminin-332 deposition (Fig. 2G; Movie 3). Approximately 67\% of mitotic spindles in basal cells were oriented parallel to or within $30^{\circ}$ of the laminin-332 layer resulting in new cells that were side-by-side (Fig. $2 \mathrm{H}$ ). The ability to measure the distribution of centrosomes and spindle orientation will allow testing of genetic, signaling and contextual determinants within basal cells that promote invasive human HG-PIN.

\section{The absence of $\alpha 6 \beta 4$ integrin expression promotes an invasive PIN phenotype}

We generated two stable RWPE-1 cell lines expressing one of two different short hairpin RNAs (shRNAs) to silence $\beta 4$ integrin (sh$\beta 4-1$ and sh- $\beta 4-2$ ) expression to test the transition to a HG-PIN phenotype. The RWPE-1 spheroids were used as these had a highly ordered laminin-332 layer that serves as a stringent test for determining the influence of $\beta 4$ integrin depletion. Western blotting confirmed efficient depletion of $\beta 4$ integrin (Fig. 3A). Spheroids made with $\beta 4$-integrin-expressing cells displayed a continuous layer of laminin-332 co-distributing with $\beta 4$ integrin [Fig. 3B,C, wild-type (WT)]. In contrast, depletion of $\beta 4$ integrin resulted in a discontinuous layer of laminin-332 (Fig. 3C, sh- $\beta 4$, arrowheads). As expected, $\beta 4$ integrin depletion did not affect the abundance of laminin-332 (Dowling et al., 1996; GeorgesLabouesse et al., 1996), as confirmed by western blotting
(Fig. 3D). Approximately $65 \%$ of $\beta 4$-integrin-depleted spheroids contained a discontinuous laminin-332 layer as compared to $19 \%$ of $\beta 4$-integrin-containing control spheroids (Fig. 3E). $\beta 4$ integrin depletion did not alter the ability of cells to form spheroids or produce laminin-332 but altered the organization of laminin-332, creating a discontinuous layer. We speculate that a discontinuous laminin-332 layer will disrupt normal glandular homeostasis and give rise to spindle misorientation to facilitate genomic instability, tissue disorganization, metastasis and expansion of cancer stem cell compartments (reviewed in Pease and Tirnauer, 2011). Therefore, this model can be used to determine whether mechanisms that disrupt laminin-332 continuity [whether it be defects of the essential adhesion receptor ( $\beta 4$ integrin), defective production of laminin332 by the stroma or loss of normal basal cell layer by luminal expansion] will stimulate the HG-PIN process.

Given that discontinuities in the basement membrane can induce cell invasion and dissemination (Nguyen-Ngoc et al., 2012), we used live imaging of $\beta 4$-integrin-depleted spheroids to investigate phenotypic changes. Strikingly, we observed prominent budding in $\beta 4$-integrin-depleted spheroids in regions of a discontinuous laminin-332 layer that was not observed in controls (Fig. 4A; Movies 4-6). The budding spheroids contained $\alpha 6$ integrin on their surfaces (Fig. 4B), which in the absence of the $\beta 4$ integrin subunit, is the $\alpha 6 \beta 1$ integrin. On day 10 , budding of the

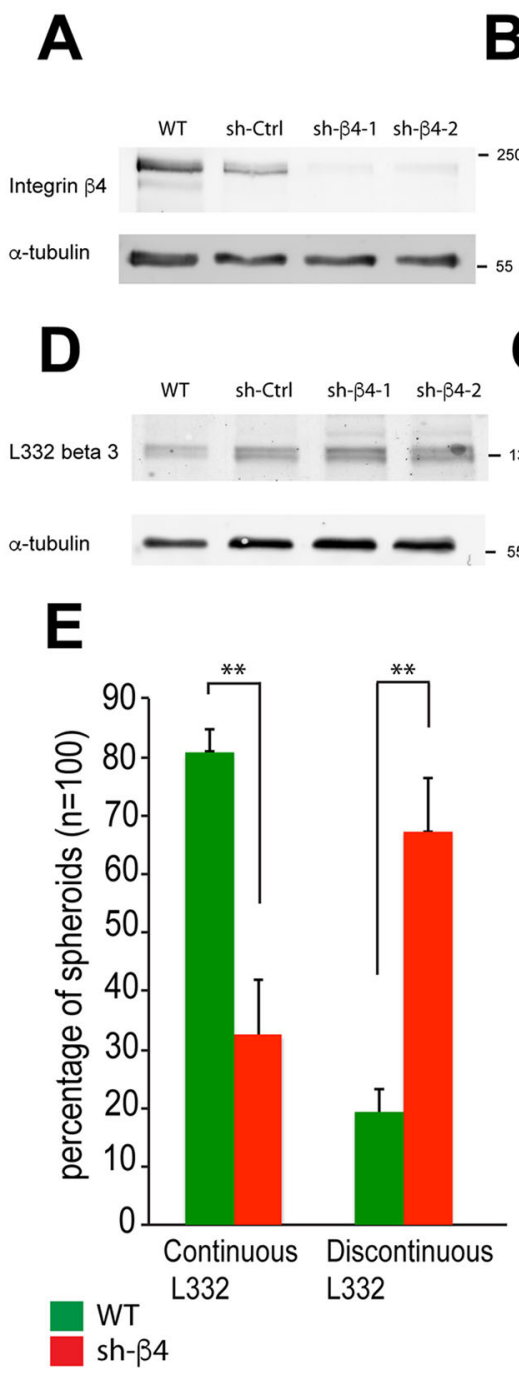

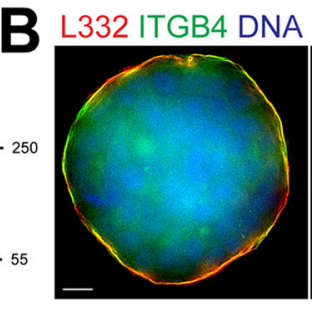
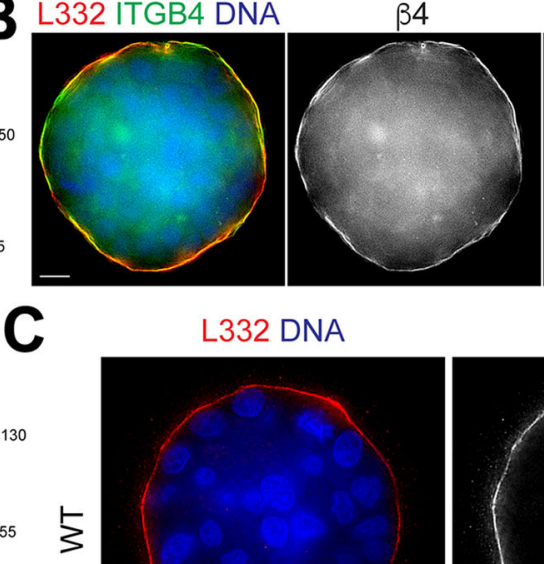

L332 DNA
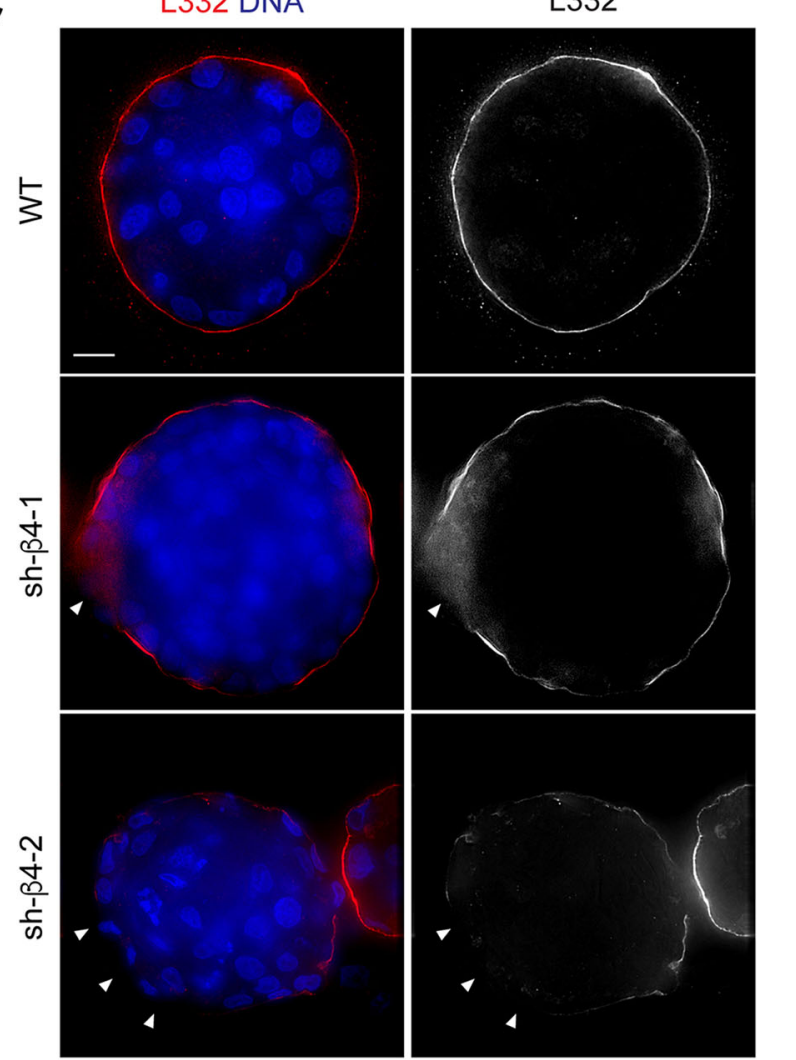

Fig. 3. 3D PIN Model - absence of $\alpha 6 \beta 4$ integrin expression and discontinuous laminin-332 layer. (A) Cell lysates from shRNA-expressing RWPE- 1 cells were probed for $\beta 4$ integrin and $\alpha$-tubulin; the position of molecular mass markers is shown in kDa. (B) Day-10 WT RWPE-1 spheroids immunostained for laminin-332 (L332, red) and $\beta 4$ integrin ( $\beta 4$, green) showing colocalization of laminin-332 and $\beta 4$ integrin (yellow). (C) Day-10 RWPE-1 spheroids immunostained for laminin332 (red) showing a continuous layer in WT RWPE-1 spheroids (top panel) and a discontinuous distribution of laminin332 (white arrowheads) in $\beta 4$

integrin-depleted spheroids (middle and bottom panels). DNA (blue). Scale bars: $10 \mu \mathrm{m}$. (D) Detection of the $\beta 3$ chain of laminin-332 and $\alpha$-tubulin in wild-type (WT), shRNA control (sh-Ctrl) and 34-integrin-depleted RWPE-1 lysates. (E) Percentage of continuous laminin332 assembly in day-10 WT and $\beta 4$ integrin-depleted spheroids. Data are mean \pm s.d. from three experiments, $n=100 .{ }^{* *} P<0.001$ (two-tailed $t$-test). 


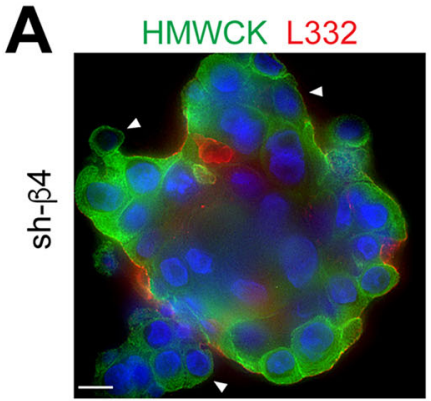

B

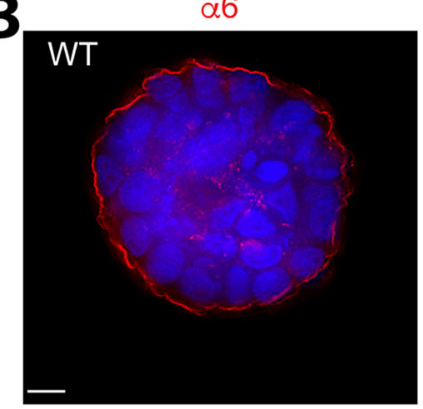

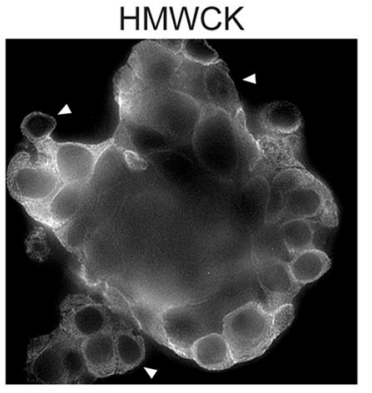

$\alpha 6$

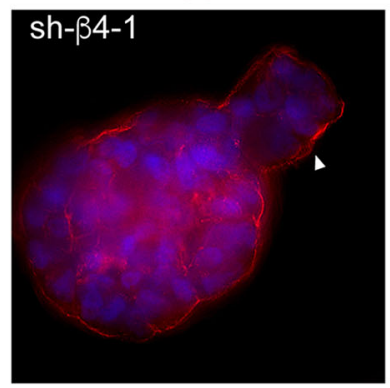

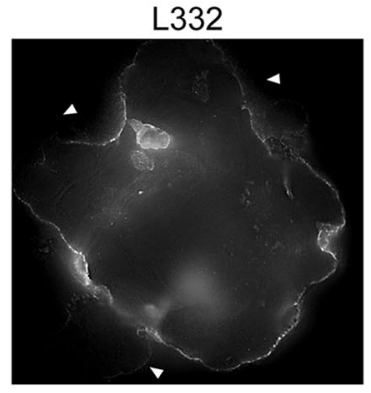

$\alpha 6$

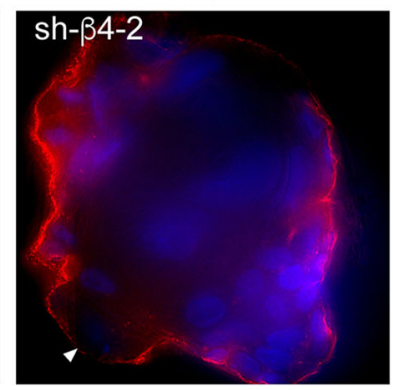

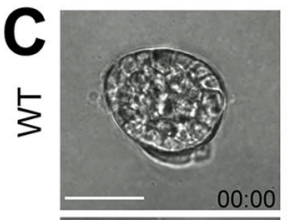
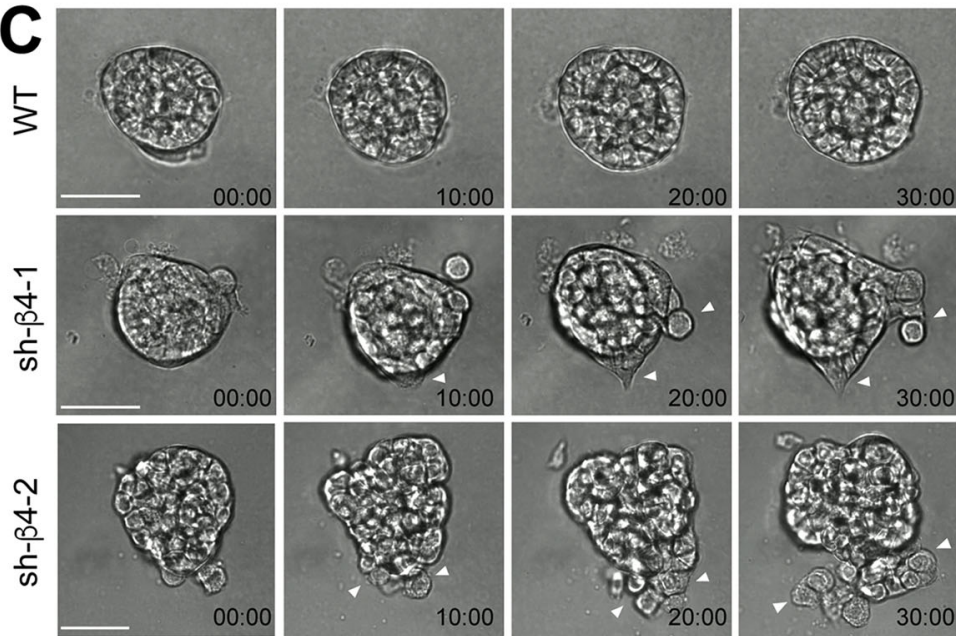
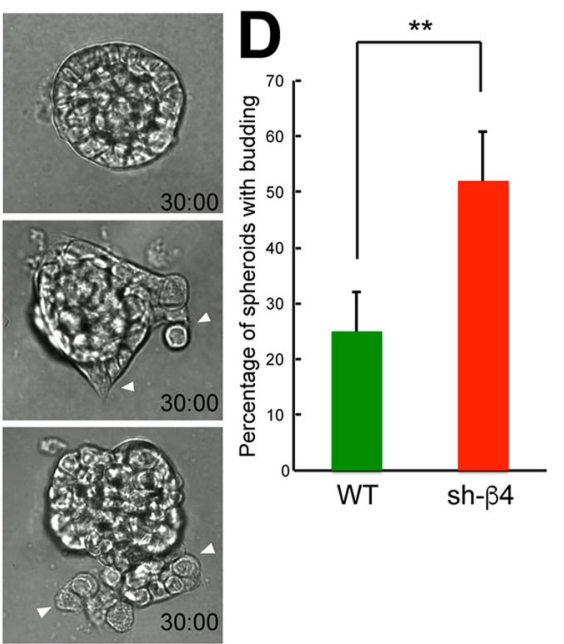

Fig. 4. Active budding of basal cells through the discontinuous laminin332 layer occurs in the absence of $\beta 4$ integrin expression in RWPE-1

spheroids. (A) Day-10 $\beta 4$ integrindepleted (sh- $\beta 4$ ) spheroid showing areas of budding (white arrowheads). Basal cells are immunolabeled for HMWCK (green) and the laminin-332 layer (L332, red). DNA, blue. Scale bar: $10 \mu \mathrm{m}$.

(B) Day-10 WT and $\beta 4$-integrin-depleted (sh- $\beta 4-1$, sh- $\beta 4-2$ ) spheroids. Cells were immunostained for $\alpha 6$ integrin ( $\alpha 6$, red). Areas of budding are indicated by white arrowheads. DNA, blue. Scale bar:

$10 \mu \mathrm{m}$. (C) Time series of WT (top row, Movie 4) and $\beta 4$-integrin-depleted spheroids (middle and bottom rows, Movies 5 and 6 ) showing examples of active cell budding time stamps as hours: minutes. Scale bars: $40 \mu \mathrm{m}$.

(D) Quantification of the percentage of spheroids with the invasive budding phenotype in WT and $\beta 4$-integrindepleted (sh- $\beta 4$ ) spheroids. Data are mean \pm s.d. from three experiments, $n=100 .{ }^{* *} P=0.0016$ (two-tailed $t$-test). epithelial cells was observed in $\beta 4$-integrin-depleted spheroids (Fig. 4C) at a significantly higher level than in WT RWPE-1 spheroids (Fig. 4D). The loss of $\beta 4$ integrin expression and persistence of $\alpha 6 \beta 1$ expression is consistent with the switching of the heterodimer composition during prostate cancer progression (Cress et al., 1995).

Taken together, our data indicate that loss of $\beta 4$ integrin expression generates laminin-332 continuity gaps through which basal cells exit the spheroid. The depletion of $\beta 4$ integrin expression in normal prostate basal cells creates a new HG-PIN-type model that can be used to determine which microenvironmental or contextual cues, and somatic mutations, promote HG-PIN progression.

\section{MATERIALS AND METHODS}

\section{Human tissue staining}

Formalin-fixed de-identified human cancer tissues were stained using a Discovery XT Automated Immunostainer (Ventana Medical Systems, Inc., Tucson, AZ) in a core support service within the UA Cancer Center. The use of de-identified human tissue was in accordance with the guidelines and approval of the University of Arizona Institutional Review Board. Informed consent was obtained for all tissue donors and that all clinical investigations have been conducted according to the principles expressed in the Declaration of Helsinki. Samples were imaged using an Olympus BX40 system (Southwest Precision Instruments, Tucson, Arizona, USA) with a 4× (NA 0.13 ) and $40 \times$ objective (NA 0.75 ).

\section{Lentiviral vectors}

The lentiviral vectors pGFP-C-shLenti shRNA-29 (Addgene, TL312080) containing a GFP reporter were used. Cells were infected with lentivirus containing the scrambled shRNA (Addgene, TR30022) as control, and shRNA hairpin against $\beta 4$ integrin. Sequences of shRNAs are $5^{\prime}$ GUACAGCGAUGACGUUCUACGCUCUCCAU-3' (sh- $\beta 4-1)$ and $5^{\prime}$ CCGUAUUGCGACUAUGAGAUGAAGGUGUG-3' (sh- $\beta 4-2$ ). Transfected cells were selected in $500 \mathrm{ng} / \mathrm{ml}$ puromycin and sorted by flow cytometry [BD Flow Machine FACSAria III; UACC Flow Cytometry shared resource (FCSR)] using the GFP signal.

\section{Epithelial non-tumorigenic human prostate cells}

RWPE-1 cells were obtained from the American Type Culture Collection (ATCC, CRL11609TM), and were maintained at $37^{\circ} \mathrm{C}$ in a $5 \% \mathrm{CO}_{2}$ atmosphere, and cultured in Iscove's modified Dulbecco's medium (IMDM; Corning, cat. no. 10-016-CV), supplemented with $10 \%$ fetal bovine serum containing detectable levels of testosterone and 
corticosteroids (FBS, Seradigm, Lot 294R13), $100 \mathrm{IU}$ penicillin, $100 \mu \mathrm{g} / \mathrm{ml}$ streptomycin and $0.25 \mu \mathrm{g} / \mathrm{ml}$ amphotericin (MP Biomedicals, cat. no. 1674049). RWPE-1 identity was verified by assessing the allelic signature of 15 different genetic markers. Immortalized human PrEC 11220 cells were generously provided by John Isaacs (The Sidney Kimmel Comprehensive Cancer Center at Johns Hopkins University, Baltimore, MD) (Dalrymple et al., 2005; Salmon et al., 2000). These cells were immortalized by sufficient basal hTERT expression. The cells have a normal human male karyotype and grow in KSFM (Life Technologies, ref. 17005-075), 100 IU penicillin, $100 \mu \mathrm{g} / \mathrm{ml}$ streptomycin and $0.25 \mu \mathrm{g} / \mathrm{ml}$ amphotericin (MP Biomedicals, cat. no. 1674049).

\section{D model staining and microscopy}

For 3D culture, the RWPE-1 cells were maintained as described previously (Tyson et al., 2007). For DHT treatment, the medium was supplemented with $5 \mathrm{nM}$ DHT (Sigma-Aldrich, D-073). Growth-factor-reduced matrigel (BD Biosciences, lot no.5173014) was used from a single lot with protein concentrations between 10 and $11 \mathrm{mg} / \mathrm{ml}$. 3D indirect immunofluorescence microscopy was performed as described previously (Debnath et al., 2002; Klebba et al., 2013). Statistical analysis was performed using Graphpad Prism 6. Antibodies used were: anti- $\alpha$-tubulin FITC-conjugated DM1a (1:100; Sigma F2168), anti-laminin-332 (1:200; Abcam ab14509), antiZO1 (1:200; Life Technologies ZO1-1A12), anti- $\beta$-catenin (1:100; Cell Signaling 9562), anti- $\beta 4$ integrin (1:200; EPR 8558), anti-HMWCK (1:200; DAKO 34ßE12), anti-p63 (1:100; Biorbyt orb214808), anti-PSA (1:100; Cell Signaling D6B1), anti-CEP135 (1:100; Abcam ab196809), and anti- $\alpha 6$ integrin (1:100; J1B5). For live-cell imaging, cells were grown on eight-well tissue culture Lab-Tek chambered coverglass (Thermo Fisher Scientific, cat. no. 155411) for 3D imaging. Cells were imaged as above under optimal growth conditions. Images were captured every $15 \mathrm{~min}$ for 2-3 days.

\section{Western blotting}

Cell extracts were produced by lysing cells in cold RIPA buffer $(50 \mathrm{mM}$ Tris- $\mathrm{HCl} \mathrm{pH} 7.4,150 \mathrm{mM} \mathrm{NaCl}, 5 \mathrm{mM}$ EDTA, $1 \% \mathrm{v} / \mathrm{v}$ Triton $\mathrm{X}-100$, $1 \% \mathrm{w} / \mathrm{v}$ sodium deoxycholate, $0.1 \% \mathrm{SDS}$ ) as described previously (Klebba et al., 2013). Antibodies used included anti- $\beta 4$ integrin (1:1000; EPR 8558), anti-laminin-332 (1:1000; Abcam ab14509), anti- $\alpha$-tubulin (1:1000; Sigma DM1a) and IRDye $800 \mathrm{CW}$ secondary antibodies (1:1500; Li-Cor Biosciences).

\section{Acknowledgements}

The gift of the normal human prostate cell line (PrEC 11220) by John T. Isaacs is gratefully acknowledged. We thank the staff of the TACMASR for the tissue processing and staining and the FCSR service for cell sorting, Dan Buster for assistance with image analysis and Cynthia S. Rubenstein for assistance with 3D culture and time-lapse microscopy. We thank William L. Harryman for the editing assistance.

\section{Competing interests}

The authors declare no competing or financial interests.

\section{Author contributions}

M.W., A.E.C. and G.C.R. designed the experiments and wrote the manuscript; M.W. performed the experiments and R.B.N. and B.S.K. contributed pathology expertise.

\section{Funding}

This work was supported in part by competitively awarded grants from the National Institutes of Health (NIH) (CA159406 to A.E.C. and P30 CA 23074). G.C.R. is grateful for support from NIH Search Results National Institute of General Medical Sciences (NIGMS) (R01GFM110166, MCB1158151), and the Phoenix Friends of the UA Cancer Center. Deposited in PMC for release after 12 months.

\section{Supplementary information}

Supplementary information available online at

http://jcs.biologists.org/lookup/doi/10.1242/jcs.188177.supplemental

\section{References}

Bello, D., Webber, M. M., Kleinman, H. K., Wartinger, D. D. and Rhim, J. S. (1997). Androgen responsive adult human prostatic epithelial cell lines immortalized by human papillomavirus 18. Carcinogenesis 18, 1215-1223.
Bonkhoff, H. (1996). Role of the basal cells in premalignant changes of the human prostate: a stem cell concept for the development of prostate cancer. Eur. Urol. 30 , 201-205

Bonkhoff, H. and Remberger, K. (1996). Differentiation pathways and histogenetic aspects of normal and abnormal prostatic growth: a stem cell model. Prostate $\mathbf{2 8}$ 98-106

Bostwick, D. G. (1996a). Progression of prostatic intraepithelial neoplasia to early invasive adenocarcinoma. Eur. Urol. 30, 145-152.

Bostwick, D. G. (1996b). Prospective origins of prostate carcinoma. Prostatic intraepithelial neoplasia and atypical adenomatous hyperplasia. Cancer $\mathbf{7 8}$ 330-336.

Bostwick, D. G., Pacelli, A. and Lopez-Beltran, A. (1996). Molecular biology of prostatic intraepithelial neoplasia. Prostate 29, 117-134.

Brown, N. H. (2011). Extracellular matrix in development: insights from mechanisms conserved between invertebrates and vertebrates. Cold Spring Harb. Perspect. Biol. 3, a005082.

Chandramouly, G., Abad, P. C., Knowles, D. W. and Lelievre, S. A. (2007). The control of tissue architecture over nuclear organization is crucial for epithelial cell fate. J. Cell Sci. 120, 1596-1606.

Cress, A. E., Rabinovitz, I., Zhu, W. and Nagle, R. B. (1995). The alpha 6 beta 1 and alpha 6 beta 4 integrins in human prostate cancer progression. Cancer Metastasis Rev. 14, 219-228.

Dalrymple, S., Antony, L., Xu, Y., Uzgare, A. R., Arnold, J. T., Savaugeot, J., Sokoll, L. J., De Marzo, A. M. and Isaacs, J. T. (2005). Role of notch-1 and Ecadherin in the differential response to calcium in culturing normal versus malignant prostate cells. Cancer Res. 65, 9269-9279.

Davis, T. L., Cress, A. E., Dalkin, B. L. and Nagle, R. B. (2001). Unique expression pattern of the alpha6beta4 integrin and laminin-5 in human prostate carcinoma. Prostate 46, 240-248.

Debnath, J., Mills, K. R., Collins, N. L., Reginato, M. J., Muthuswamy, S. K. and Brugge, J. S. (2002). The role of apoptosis in creating and maintaining luminal space within normal and oncogene-expressing mammary acini. Cell 111, 29-40.

Dowling, J., Yu, Q. C. and Fuchs, E. (1996). Beta4 integrin is required for hemidesmosome formation, cell adhesion and cell survival. J. Cell Biol. 134 559-572.

Ekblom, M., Falk, M., Salmivirta, K., Durbeej, M. and Ekblom, P. (1998). Laminin isoforms and epithelial development. Ann. N. Y. Acad. Sci. 857, 194-211.

Georges-Labouesse, E., Messaddeq, N., Yehia, G., Cadalbert, L., Dierich, A. and Le Meur, M. (1996). Absence of integrin alpha 6 leads to epidermolysis bullosa and neonatal death in mice. Nat. Genet. 13, 370-373.

Haffner, M. C., Weier, C., Xu, M. M., Vaghasia, A., Gurel, B., Gumuskaya, B., Esopi, D. M., Fedor, H., Tan, H.-L., Kulac, I. et al. (2016). Molecular evidence that invasive adenocarcinoma can mimic prostatic intraepithelial neoplasia (PIN) and intraductal carcinoma through retrograde glandular colonization. J. Pathol 238, 31-41.

Haggman, M. J., Macoska, J. A., Wojno, K. J. and Oesterling, J. E. (1997). The relationship between prostatic intraepithelial neoplasia and prostate cancer: critical issues. J. Urol. 158, 12-22.

Hao, J., Yang, Y., McDaniel, K. M., Dalkin, B. L., Cress, A. E. and Nagle, R. B. (1996). Differential expression of laminin 5 (alpha 3 beta 3 gamma 2) by human malignant and normal prostate. Am. J. Pathol. 149, 1341-1349.

Hudson, D. L. (2004). Epithelial stem cells in human prostate growth and disease. Prostate Cancer Prostatic Dis. 7, 188-194.

Iwata, T., Schultz, D., Hicks, J., Hubbard, G. K., Mutton, L. N., Lotan, T. L., Bethel, C., Lotz, M. T., Yegnasubramanian, S., Nelson, W. G. et al. (2010) MYC overexpression induces prostatic intraepithelial neoplasia and loss of Nkx3.1 in mouse luminal epithelial cells. PLoS ONE 5, e9427.

Karthaus, W. R., laquinta, P. J., Drost, J., Gracanin, A., van Boxtel, R. Wongvipat, J., Dowling, C. M., Gao, D., Begthel, H., Sachs, N. et al. (2014). Identification of multipotent luminal progenitor cells in human prostate organoid cultures. Cell 159, 163-175.

Klebba, J. E., Buster, D. W., Nguyen, A. L., Swatkoski, S., Gucek, M., Rusan, N. M. and Rogers, G. C. (2013). Polo-like kinase 4 autodestructs by generating its Slimb-binding phosphodegron. Curr. Biol. 23, 2255-2261.

Kumaresan, K., Kakkar, N., Verma, A., Mandal, A. K., Singh, S. K. and Joshi, K. (2010). Diagnostic utility of alpha-methylacyl CoA racemase (P504S) \& HMWCK in morphologically difficult prostate cancer. Diagn. Pathol. 5, 83.

Montironi, R. and Schulman, C. C. (1996). Precursors of prostatic cancer: progression, regression and chemoprevention. Eur. Urol. 30, 133-137.

Montironi, R., Bostwick, D. G., Bonkhoff, H., Cockett, A. T. K., Helpap, B., Troncoso, P. and Waters, D. (1996a). Origins of prostate cancer. Cancer 78 362-365

Montironi, R., Pomante, R., Diamanti, L., Hamilton, P. W., Thompson, D. and Bartels, P. H. (1996b). Evaluation of prostatic intraepithelial neoplasia after treatment with a 5-alpha-reductase inhibitor (finasteride). A methodologic approach. Anal. Quant. Cytol. Histol. 18, 461-470.

Mosquera, J.-M., Perner, S., Genega, E. M., Sanda, M., Hofer, M. D., Mertz, K. D. Paris, P. L., Simko, J., Bismar, T. A., Ayala, G. et al. (2008). Characterization of TMPRSS2-ERG fusion high-grade prostatic intraepithelial neoplasia and potentia clinical implications. Clin. Cancer Res. 14, 3380-3385. 
Mosquera, J.-M., Mehra, R., Regan, M. M., Perner, S., Genega, E. M., Bueti, G., Shah, R. B., Gaston, S., Tomlins, S. A., Wei, J. T. et al. (2009). Prevalence of TMPRSS2-ERG fusion prostate cancer among men undergoing prostate biopsy in the United States. Clin. Cancer Res. 15, 4706-4711.

Nagle, R. B. and Cress, A. E. (2011). Metastasis update: human prostate carcinoma invasion via tubulogenesis. Prostate Cancer 2011, 249290.

Nagle, R. B., Petein, M., Brawer, M., Bowden, G. T. and Cress, A. E. (1992). New relationships between prostatic intraepithelial neoplasia and prostatic carcinoma. J. Cell. Biochem. Suppl. 16H, 26-29.

Nagle, R. B., Knox, J. D., Wolf, C., Bowden, G. T. and Cress, A. E. (1994) Adhesion molecules, extracellular matrix, and proteases in prostate carcinoma. J. Cell. Biochem. Suppl. 19, 232-237.

Nagle, R. B., Hao, J., Knox, J. D., Dalkin, B. L., Clark, V. and Cress, A. E. (1995). Expression of hemidesmosomal and extracellular matrix proteins by normal and malignant human prostate tissue. Am. J. Pathol. 146, 1498-1507.

Nguyen-Ngoc, K.-V., Cheung, K. J., Brenot, A., Shamir, E. R., Gray, R. S., Hines, W. C., Yaswen, P., Werb, Z. and Ewald, A. J. (2012). ECM microenvironment regulates collective migration and local dissemination in normal and malignant mammary epithelium. Proc. Natl. Acad. Sci. USA 109, E2595-E2604.

Pease, J. C. and Tirnauer, J. S. (2011). Mitotic spindle misorientation in cancer-out of alignment and into the fire. J. Cell Sci. 124, 1007-1016.

Petein, M., Michel, P., van Velthoven, R., Pasteels, J.-L., Brawer, M. K., Davis, J. R., Nagle, R. B. and Kiss, R. (1991). Morphonuclear relationship between prostatic intraepithelial neoplasia and cancers as assessed by digital cell image analysis. Am. J. Clin. Pathol. 96, 628-634.

Pontes-Junior, J., Reis, S. T., Dall'Oglio, M., Neves de Oliveira, L. C., Cury, J., Carvalho, P. A., Ribeiro-Filho, L. A., Moreira Leite, K. R. and Srougi, M. (2009) Evaluation of the expression of integrins and cell adhesion molecules through tissue microarray in lymph node metastases of prostate cancer. J. Carcinog. 8, 3.

Pulkkinen, L. and Uitto, J. (1998). Hemidesmosomal variants of epidermolysis bullosa. Mutations in the alpha6beta4 integrin and the $180-\mathrm{kD}$ bullous pemphigoid antigen/type XVII collagen genes. Exp. Dermatol. 7, 46-64.

Roh, M., Franco, O. E., Hayward, S. W., van der Meer, R. and Abdulkadir, S. A (2008). A role for polyploidy in the tumorigenicity of Pim-1-expressing human prostate and mammary epithelial cells. PLoS ONE 3, e2572.
Salmon, P., Oberholzer, J., Occhiodoro, T., Morel, P., Lou, J. and Trono, D. (2000). Reversible immortalization of human primary cells by lentivector-mediated transfer of specific genes. Mol. Ther. 2, 404-414

Schmelz, M., Moll, R., Hesse, U., Prasad, A. R., Gandolfi, J. A., Hasan, S. R., Bartholdi, M. and Cress, A. E. (2005). Identification of a stem cell candidate in the normal human prostate gland. Eur. J. Cell Biol. 84, 341-354.

Thomson, A. A. and Marker, P. C. (2006). Branching morphogenesis in the prostate gland and seminal vesicles. Differentiation 74, 382-392.

Tyson, D. R., Inokuchi, J., Tsunoda, T., Lau, A. and Ornstein, D. K. (2007). Culture requirements of prostatic epithelial cell lines for acinar morphogenesis and lumen formation in vitro: role of extracellular calcium. Prostate 67, 1601-1613.

Verhagen, A. P. M., Aalders, T. W., Ramaekers, F. C. S., Debruyne, F. M. J. and Schalken, J. A. (1988). Differential expression of keratins in the basal and luminal compartments of rat prostatic epithelium during degeneration and regeneration. Prostate 13, 25-38.

Verhagen, A. P., Ramaekers, F. C., Aalders, T. W., Schaafsma, H. E., Debruyne, F. M. and Schalken, J. A. (1992). Colocalization of basal and luminal cell-type cytokeratins in human prostate cancer. Cancer Res. 52, 6182-6187.

Wang, X.-S., Shankar, S., Dhanasekaran, S. M., Ateeq, B., Sasaki, A. T., Jing, X., Robinson, D., Cao, Q., Prensner, J. R., Yocum, A. K. et al. (2011). Characterization of KRAS rearrangements in metastatic prostate cancer Cancer Discov. 1, 35-43.

Wang, H., Lacoche, S., Huang, L., Xue, B. and Muthuswamy, S. K. (2013). Rotational motion during three-dimensional morphogenesis of mammary epithelial acini relates to laminin matrix assembly. Proc. Natl. Acad. Sci. USA 110, 163-168.

Webber, M. M., Bello, D., Kleinman, H. K. and Hoffman, M. P. (1997). Acinar differentiation by non-malignant immortalized human prostatic epithelial cells and its loss by malignant cells. Carcinogenesis 18, 1225-1231.

Wilhelmsen, K., Litjens, S. H. M. and Sonnenberg, A. (2006). Multiple functions of the integrin alpha6beta4 in epidermal homeostasis and tumorigenesis. Mol. Cell. Biol. 26, 2877-2886.

Xia, J., Swiercz, J. M., Banon-Rodriguez, I., Matkovic, I., Federico, G., Sun, T., Franz, T., Brakebusch, C. H., Kumanogoh, A., Friedel, R. H. et al. (2015) Semaphorin-Plexin signaling controls mitotic spindle orientation during epithelial morphogenesis and repair. Dev. Cell 33, 299-313.

Yurchenco, P. D. (2015). Integrating activities of laminins that drive basement membrane assembly and function. Curr. Top. Membr. 76, 1-30. 